\title{
钻钛矿型 $\mathrm{AMF}_{3}$ 中 $\mathrm{Eu}^{2+}$ 的光谱结构 与其取代格位 ${ }^{*}$
}

石春山 叶泽人 胡宁海 周清廉

(中国科学院长春应用化学研究所)

近年来,钻钛矿结构的复合氧化物 $\mathrm{AMF}_{3}(\mathrm{~A}=\mathrm{Na}, \mathrm{K}, \mathrm{Rb}, \mathrm{Cs} ; \mathrm{M}=\mathrm{Be}, \mathrm{Mg}, \mathrm{Ca}, \mathrm{Zn}$ 等)， 由于容易掺人二价或三价激活离子, 所以作为可调谐固体激光晶体的基质材料引起人们极大 关注. 至今,已在许多这类化合物中观察到了某些激活离子的激光振荡 ${ }^{[1,2]}$. 尤其是掺 $\mathrm{Cr}^{3+}$ 的 $\mathrm{KZnF}_{3}$ 终端声子激光器已实现了室温运转 ${ }^{[3]}$.

$\mathrm{Eu}^{2+}$ 是重要的稀土激活离子. $f \rightarrow d$ 跃迁在可见区有较强的吸收, 用作激光材料有利于 提高原浦效率; 用作发光材料可以利用基质晶格的影响, 任意选择发射波长. $\mathrm{Eu}^{2+}$ 的特征发 射是宽带, 但在许多 $\mathrm{AMF}_{3}$ 中 $\mathrm{Eu}^{2+}$ 的荧光光谱都有尖峰出现. 对于光谱结构上的这种特殊 性,被认为是由于 $\mathrm{Eu}^{2+}$ 对基质中阳离子的格位取代不同引起的 ${ }^{[4]}$.

实验证明, $\mathrm{Eu}^{2+}$ 的电子跃迁发射 $(d \rightarrow f$ 或 $f \rightarrow f$ ) 类型, 强烈取决于其对基质阳离子的 取代格位. 这种关系对研究 $\mathrm{Eu}^{2+}$ 光谱性质与基质晶体结构之间的内在联系, 以及探找具有特 定发射波长的新基质材料都具有重要意义。

本工作主要以掺 $\mathrm{Eu}^{2+}$ 的 $\mathrm{KMgF}_{3}$ 为例, 实验确证 $\mathrm{Eu}^{2+}$ 在基质中的取代格位, 以及与其 荻光光谱结构的关系.

\section{一、理 论判据}

$\mathrm{KMgF}_{3}$, 属立方晶系. $\mathrm{F}^{-}$位于面心; $\mathrm{Mg}^{2+}$ 位于体心, 八配位; $\mathrm{K}^{+}$处在立方体顶角, 十二 配位. Abrahan 等 ${ }^{[5]}$ 曾经指出,对于掺二价稀土离子 $\left(\mathrm{R}^{2+}\right)$ 的 $\mathrm{KMgF}_{3}$ 来说, 如果该离子是定 域在十二配位的格位中, 就是说, $\mathrm{R}^{2+}$ 取代了 +1 价阳离子 $\mathrm{K}^{+}$, 则晶场参数 $\mathrm{B}_{4}$ 和 $\mathrm{B}_{6}$ 均应为负 值. 如果 $R^{2+}$ 是定域在八配位的格位中, 即 $R^{2+}$ 取代 +2 价阳离子 $\mathrm{Mg}^{2+}$, 则 $\mathrm{B}_{4}$ 和 $\mathrm{B}_{6}$ 均应为: 正值.

Альтшулер 等 ${ }^{[6]}$ 对 $\mathrm{Eu}^{2+}$ 在 $\mathrm{KMgF}_{3}$ 中所处格位作了晶场参数计算. 立方场中 $\mathrm{Eu}^{2+}$ 的 哈密顿函数可以写作

$$
\begin{aligned}
H= & 16 B_{4} \sqrt{\frac{6}{11}}\left\{\sqrt{\frac{7}{12}} u_{0}^{4}+\sqrt{\frac{5}{25}}\left(u_{4}^{6}+u_{-4}^{6}\right)\right\} \\
& +320 B_{6} \sqrt{\frac{14}{429}}\left\{-\sqrt{\frac{1}{8}} u_{0}^{6}+\sqrt{\frac{7}{16}}\left(u_{4}^{6}+u_{-4}^{6}\right)\right\},
\end{aligned}
$$

利用 $(\mathrm{A})$ 式将 $\mathrm{Eu}^{2+}$ 离子 $6 p$ 态的矩阵元写作

本文 1986 年 12 月 23 日收到. 1987 年 4 月 3 日收到借改程.

- 国家目然科学基金资助项目。 


$$
\begin{aligned}
& \left\langle f^{n} J_{i}^{a} \Gamma_{i}\left|H_{c r}\right| f^{n} J_{m}^{\beta} \Gamma_{i}\right\rangle-\delta\left(\Gamma_{i}, \Gamma_{i}\right)\left\{16 B_{4} \sqrt{\frac{6}{11}}\right. \\
& \text { - }\left(4 \Gamma_{1}, J_{m}^{\beta} \Gamma_{i} \mid J_{i}^{a} \Gamma_{i}\right) \beta_{l m}+320 B_{6} \sqrt{\frac{14}{429}}\left(6 \Gamma_{1}, J_{m}^{\beta} \Gamma_{i} \mid\right. \\
& \text { - } \left.\left.J_{i}^{a} \Gamma_{i}\right) r_{l m}\right\},
\end{aligned}
$$

(B) 式经简化处理后,最终得到 $\mathrm{KMgF}_{3}$ 中 $\mathrm{Eu}^{2+}$ 周围晶场参数均为负值,即

$$
B_{4}=-46, B_{6}=-25 \text {. }
$$

依照 Abrahan 的判据推定，在 $\mathrm{KMgF}_{3}$ 中 $\mathrm{Eu}^{2+}$ 应当是定域在十二配位的 $\mathrm{K}^{+}$格位，而不是 $\mathrm{Mg}^{2+}$ 格位. 作者利用这一判定结果成功地解释了 $\mathrm{KMgF}_{3}$ 中 $\mathrm{Eu}^{2+}$ 的光谱特性 ${ }^{[6]}$. 但是, 这 一理论判据至今尚未得到实验确证.

\section{二、实验及结果分析}

\section{1. 用不同量的 $\mathrm{Be}^{2+}, \mathrm{Ca}^{2+}$ 分别取代 $\mathrm{KMgF}_{3}$ 中 $\mathrm{Mg}^{2+}$ ，考察 $\mathrm{Ea}^{2+}$ 的苂光光诣结构变} 代的值动碱土金属中 $\mathrm{Be}^{2+}$ 的有效离子半径 (Shannon 半径)最小, 根据鲍林化学键 速论分析, $\mathrm{Be}-\mathrm{F}$ ，键的键强应大于 $\mathrm{Mg}-\mathrm{F}$ 键. 就是说, $\mathrm{BeF}_{2}$ 比 $\mathrm{MgF}_{2}$ 应具有更强的共价性.

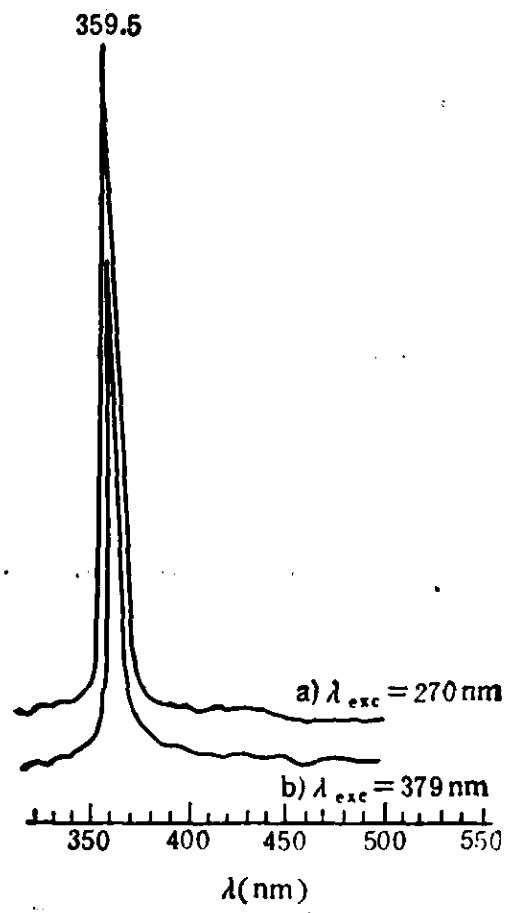

$1 \mathrm{KMF}_{3}$ 中 $\mathrm{Eu}^{2+}$ 的苂光光谱 $(300 \mathrm{~K})$
a) $\mathrm{M}=\mathrm{Be}$;
b) $\mathrm{M}=\mathrm{Mg}$
(图中 $379 \mathrm{~nm}$ 应为 $312 \mathrm{~nm}$ )

按鲍林公式 ${ }^{[7]}$

$$
P=\Delta 16+\Delta^{23.5} \text { ( } \Delta \text { 为原子电负性差) }
$$

计算, $\mathrm{BeF}_{2}$ 和 $\mathrm{MgF}_{2}$ 的离子性百分比 $(P)$ 分别为

$$
P_{\mathrm{BeP}_{2}} \doteq 60 \%, P_{\mathrm{MgP}_{2}} \doteq 70 \% \text {. }
$$

用不同量 $\mathrm{Be}^{2+}$ 逐渐取代掺 $\mathrm{Eu}^{2+}$ 的 $\mathrm{KMgF}_{3}$ 中 $\mathrm{Mg}^{2+}$, 直至完全取代,形成 $\mathrm{KBeF}_{3}$ 。假若 $\mathrm{Eu}^{2+}$ 是占据 着 $\mathrm{Mg}^{2+}$ 格位,那么随着 $\mathrm{Be}^{2+}$ 对 $\mathrm{Mg}^{2+}$ 的不断取代，处 于 $\mathrm{Mg}^{2+}$ 格位的 $\mathrm{Eu}^{2+}$ 在其光谱特征上应当逐渐产生 变化, 即随 $\mathrm{Be}^{2+}$ 增加,共价性增强, $\mathrm{Eu}^{2+}$ 的 $5 d$ 能带重 心下降，发射蜂位应向长波方向移动. 对碱土金属系 讧化合物而言，按照这一规律， $\mathrm{KBeF}_{3}$ 中 $\mathrm{Eu}^{2+}$ 的发射 位置应处于最长波位置。

但是实验结果与此相反. $\mathrm{KBeF}_{3}$ 中 $\mathrm{Eu}^{2+}$ 的发射 峰位置与 $\mathrm{KMgF}_{3}$ 中一样, 而且也呈线状(图 1). 这一 反证结果可以表明, $\mathrm{KMgF}_{3}$ 中 $\mathrm{Eu}^{2+}$ 不是处于 $\mathrm{Mg}^{2+}$ 格位.

同理，由于 $\mathrm{Ca}^{2+}$ 的有效离子半径比 $\mathrm{Mg}^{2+}$ 大，

$\mathrm{Ca}-\mathrm{F}$ 键的键强应比 $\mathrm{Mg}-\mathrm{F}$ 键弱, 即 $\mathrm{CaF}_{2}$ 的共价性应比 $\mathrm{MgF}_{2}$ 弱 $\left(P_{\mathrm{Ca}_{2}} \doteq 80 \%\right)$.

用不同量 $\mathrm{Ca}^{2+}$ 逐渐取代 $\mathrm{KMgF}_{3}$ 中 $\mathrm{Mg}^{2+}$ ，直至完全取代，形成 $\mathrm{KCaF}_{3}$ 。假定 $\mathrm{Eu}^{2+}$ 是占 据 $\mathrm{Mg}^{2+}$ 格位,那么按照上述规律, $\mathrm{Eu}^{2+}$ 的发射波长应随 $\mathrm{Ca}^{2+}$ 对 $\mathrm{Mg}^{2+}$ 逐渐取代而向短波方 向移动.

但实验结果相反. 如表 1 所示,由于 $\mathrm{Ca}^{2+}$ 对 $\mathrm{Mg}^{2+}$ 的取代, $\mathrm{Eu}^{2+}$ 荻光光谱结构中除尖峰 外,在长波方向又出现有宽带,并且随 $\mathrm{Ca}^{2+}$ 取代量的不断增加, $\mathrm{KMgF}_{3}$ 中 $\mathrm{Eu}^{2+}$. 的(线/带)强 
表 $1 \mathrm{Eu}^{2+}$ 在 $\mathrm{KMF}_{3}(\mathrm{M}=\mathrm{Be}, \mathrm{Mg}, \mathrm{Ca})$ 中光谱结构 (300K)

\begin{tabular}{|c|c|c|c|c|}
\hline \multirow{2}{*}{ 基 质 组 成 } & \multirow{2}{*}{$\begin{array}{c}\text { 激发波长 } \\
\lambda / \mathrm{nm}\end{array}$} & \multicolumn{2}{|c|}{ 发射波长， $\lambda / \mathrm{n}$ 血 } & \multirow{2}{*}{$\begin{array}{l}\text { 线/带强度比， } \\
\text { (面比) }\end{array}$} \\
\hline & & $t \rightarrow t$ & $d \rightarrow f$ & \\
\hline $\mathrm{KMgF}_{3}$ & 312 & 359.5 & - & 极大 \\
\hline $\mathrm{KMg}_{0.8} \mathrm{Be}_{0.2} \mathrm{~F}_{3}$ & 295 & 359.5 & - & 极大 \\
\hline 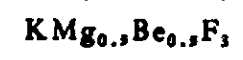 & 280 & 359.5 & - & 极大 \\
\hline $\mathrm{KMg}_{\mathbf{O}_{0.2}} B \mathrm{e}_{0.8} \mathrm{~F}_{3}$ & 270 & 359.5 & - & 极大 \\
\hline $\mathrm{KBeF}_{3}$ & 270 & 359.5 & - & 极大 \\
\hline $\mathrm{KMg}_{0.8} \mathrm{Ca}_{\mathrm{a}_{0.2} \mathrm{~F}_{3}}$ & 307 & 359.5 & 425 & 4.00 \\
\hline 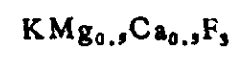 & 276 & 359.5 & 425 & 1.60 \\
\hline $\mathrm{KMg}_{0.2} \mathrm{C}_{\mathrm{s}_{0.0} \mathrm{~F}}$ & 276 & 359.5 & 425 & 0.32 \\
\hline $\mathrm{KCaF}_{3}$ & 379 & - & 425 & 0 \\
\hline
\end{tabular}

度比逐渐变小, 即尖峰发射强度逐渐下降, 而带状峰相应增强, 直到形成 $\mathrm{KCaF}_{3}$ 尖峰完全消 失, 只旺现出 $\mathrm{Eu}^{2+}$ 的特征宽带发射,最大中心位置在 $425 \mathrm{~nm}$.

随着 $\mathrm{Mg}^{2+}$ 不断被 $\mathrm{Ca}^{2+}$ 取代, 由 $\mathrm{KMgF}_{3}$ 逐 渐转变成 $\mathrm{KCaF}_{3}$ 。在这一过程中,由于 $\mathrm{K}^{+}$和 $\mathrm{Ca}^{2+}$ 的原子电负性都比较小 (近于 $\mathrm{Eu}^{2+}$ 的电负性), 所 以对于 $\mathrm{Eu}^{2+}$ 的取代来说，基质中两种阳离子的 格位都有可能被占据. 但就离子半径而言, $\mathrm{Ca}^{2+}$ 更利于被 $\mathrm{Eu}^{2+}$ 取代. 当 $\mathrm{Eu}^{2+}$ 占据 $\mathrm{Ca}^{2+}$ 离子格 位时,与其占据 $\mathrm{K}^{+}$格位时相比，所受晶场环境的 影晌更会增强, $\mathrm{Eu}^{2+}$ 的 $5 d$ 能级䢃裂会加大。于 是使激发和发射的谱带明显加宽, 呈带状 (图 2).

这一反证结果, 也表明 $\mathrm{Eu}^{2+}$ 不占据 $\mathrm{Mg}^{2+}$ 格 位.

由上述光谱结构中我们可以确认, $\mathrm{ABeF}_{3}$ 和 $\mathrm{AMgF}_{3}(\mathrm{~A}=\mathrm{Na}, \mathrm{K}, \mathrm{Rb}, \mathrm{Cs})$ 中 $\mathrm{Eu}^{2+}$ 不占据+2 价阳离子格位; 在 $\mathrm{ACaF}_{3}$ 中 $\mathrm{Eu}^{2+}$ 不占据 +1 价 阳离子格位. 如果以 $\mathrm{Ni}^{2+}$ 作激活离子, 则 ESR 数据证明, 上述各体系中 $\mathrm{Ni}^{2+}$ 均取代基质中 +2 价阳离子格位 ${ }^{[8]}$.

\section{2 对 $\mathrm{Eu}, \mathrm{K}, \mathrm{Mg}$ 在 $\mathrm{KMgF}_{3}$ 单晶中格位占有} 率作传正，观察 $\mathrm{Eu}$ 占据的格位坩埚下降法、 昰气氛中生长的掺 $\mathrm{Eu}^{2+} \mathrm{KMgF}_{3}$ 单晶. 采用 $R 3$

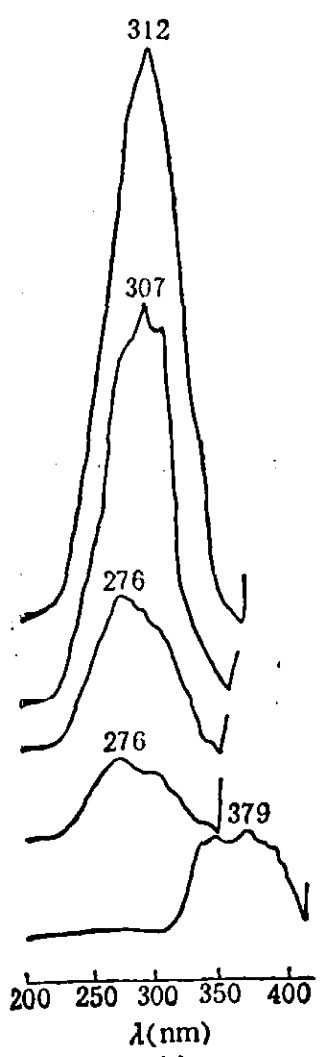

(a)

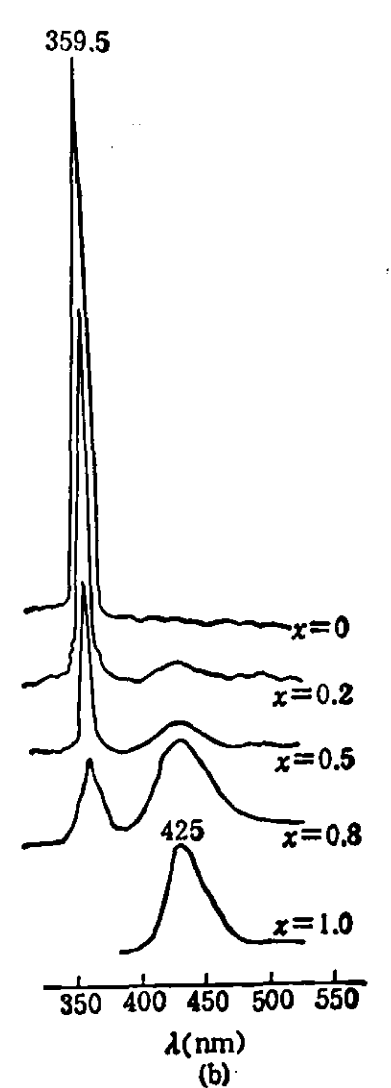

(b)

图 $2 \mathrm{KM}_{1-x} \mathrm{Ca}_{x} \mathrm{~F}_{3}$ 中 $\mathrm{Eu}^{2+}$ 的光谱 $(300 \mathrm{~K})$
(a) 激发;
(b) 发射

$m / e$ 型四圆衍射仪 ( $\mathrm{Mo}, \mathrm{K} \alpha$ 辐射, $\theta / 2 \theta$ 扫描方式), 通过 52 个衍射点对 $\mathrm{KMgF}_{3}$ 中 $\mathrm{Eu}^{2+}$ 在 $\mathrm{K}, \mathrm{Mg}$ 格位上占有率作了搜集 $(I>1.5 \sigma(I))$.

设定 $\mathrm{Eu}^{2+}$ 占据 $\mathrm{Mg}^{2+}$ 格位,不占据 $\mathrm{K}^{+}$格位. 用最小二乘方法对位置占有率从 0.5 和 1.0 分别开始修正. 结果, 得不到收玫的偏离因子 $(R)$, 表明 $\mathrm{Eu}^{2+}$ 未占据 $\mathrm{Mg}^{2+}$ 格位.

再设定 $\mathrm{Eu}^{2+}$ 占据 $\mathrm{K}^{+}$格位,不占据 $\mathrm{Mg}^{2+}$ 格位. 仍用最小二乘方法对位置占有率分别从 
0 和 0.5 开始修正. 结果,当 $\mathrm{Eu}^{2+}$ 和 $\mathrm{K}^{+}$占有率分别为 10\% 和 $\sim 90 \%$ 时, 即

$$
\mathrm{Eu}(S \cdot O \cdot f)=0.10, \mathrm{~K}(S \cdot O \cdot f)-0.90
$$

时,偏离因子 $R$ 得到较好收敛

$$
R=\frac{\Sigma|| F_{0}|-| F_{c} \|}{\Sigma\left|F_{0}\right|}=0.0298 .
$$

这一结果与化学方法掺杂浓度相符. 由此定量地证明了 $\mathrm{Eu}^{2+}$ 在 $\mathrm{KMgF}$. 中占据 $\mathrm{K}^{+}$格位, 未 占据 $\mathrm{Mg}^{2+}$ 格位。

光谱数据表明,上述结论均可推用到掺 $\mathrm{Eu}^{2+}$ 的所有钙针矿结构 $\mathrm{AMF}_{3}(\mathrm{~A}-\mathrm{Na}, \mathrm{K}, \mathrm{Rb}$ ， Cs; $\mathrm{M}-\mathrm{Be}, \mathrm{Mg}, \mathrm{Ca}$ ) 中去.

\section{三、结 束 语}

通过实验确立了 $\mathrm{Eu}^{2+}$ 在 $\mathrm{AMF}_{3}$ 中荧光光谱结构与其取代格位的关系. $\mathrm{ACaF}_{3}$ 中 $\mathrm{Eu}^{2+}$ 占 据 $\mathrm{Ca}^{2+}$ 格位,荧光光谱是宽带; $\mathrm{AMgF}_{3}$ 和 $\mathrm{ABeF}_{3}$ 中 $\mathrm{Eu}^{2+}$ 占据 $\mathrm{A}^{+}$格位, 荧光光谱呈窄带尖 发射。反之,由这些不同发射特征也可判断出 $\mathrm{Eu}^{2+}$ 在 $\mathrm{AMF}_{3}$ 中的取代格位.

\section{考文 解}

[1] Powell, R. C. et al., Optics Letzers, 10(1985), 5: 212-214.

[2] Brauch, U. et al., Optics Commun., 55(1985), 1: 35-40.

[3] Dorr, U., Optics Commun, 49(1984), 1: 61-64.

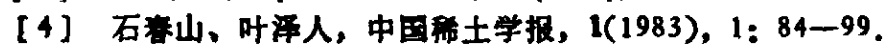

[5] Abrahan, M. M. et al, Phys. Rev, B, 3(1971), 9: 2855-2864.

[6] Альпиулер Н. С., Д Др., Физика Твёрдого Тела, 15 (1973), 8: 2407-2411.

[7] Nassau, K., in Applied Solid Szaze Science (Ed. Wolfe, R.), Vol. 2, Academic Press, New York and London, 197 . 101-105.

[8] Kerkouri, N. et al., Maser. Res. Bull., 19(1984), 2: 751-757. 